\title{
ON THE NUMBER OF DIVISORS OF $n$ ! AND OF THE FIBONACCI NUMBERS
}

\author{
Florian Luca and Paul Thomas Young
}

UNAM, Mexico, University of Witwatersrand, South Africa and College of Charleston, USA

ABstract. Let $d(m)$ be the number of divisors of the positive integer $m$. Here, we show that if $n \notin\{3,5\}$, then $d(n !)$ is a divisor of $n$ !. We also show that the only positive integers $n$ such that $d\left(F_{n}\right)$ divides $F_{n}$, where $F_{n}$ is the $n$th Fibonacci number, are $n \in\{1,2,3,6,24,48\}$.

\section{INTRODUCTION}

Let $d(m)$ be the number of divisors of the positive integer $m$. The number of divisors of $n$ ! was studied in the paper [5]. The equation $d(n !)=m$ ! was studied in [6]. More generally, the fractions $d(n !) / m$ ! were studied in [1]. Here, we look at positive integers $n$ such that $d(n !)$ is a divisor of $n$ !. Positive integers $m$ such that $d(m)$ divides $m$ were studied in [8].

Our first result is the following.

THEOREM 1.1. If $n \geqslant 6$, then $d(n !)$ is a divisor of $n$ !.

Let $\left\{F_{n}\right\}_{n \geqslant 1}$ be the Fibonacci sequence given by $F_{1}=F_{2}=1$ and $F_{n+2}=$ $F_{n}+F_{n+1}$ for all $n \geqslant 1$. Our result is the following.

THEOREM 1.2. The only positive integers $n$ such that $d\left(F_{n}\right)$ divides $F_{n}$ are $n \in\{1,2,3,6,24,48\}$.

For a positive real number $x$, we write $\pi(x)$ for the number of primes $p \leqslant x$.

2010 Mathematics Subject Classification. 11N37, $11 \mathrm{~B} 39$.

Key words and phrases. Divisors, factorials, Fibonacci numbers. 


\section{Proof of Theorem 1.1}

We first ran computations with Mathematica and with PARI which verified that $d(n$ !) $\mid n$ ! for all $n<3400$ except for $n=3$, 5; this verification takes only a few minutes of computational time. From now on, we assume that $n \geqslant 3400$.

We write

$$
n !=\prod_{p \leqslant n} p^{a_{p}(n)}
$$

It is then well-known that

$$
a_{p}(n)=\frac{n-s_{p}(n)}{p-1},
$$

where $s_{p}(n)$ is the sum of the digits of $n$ written in base $p$. Clearly,

$$
1 \leqslant s_{p}(n) \leqslant(p-1)\left(\left\lfloor\frac{\log n}{\log p}\right\rfloor+1\right) \text { for all primes } p \leqslant n .
$$

Then

$$
d(n !)=\prod_{p \leqslant n}\left(a_{p}(n)+1\right) .
$$

The method of proof consists in finding an injection $f:\{p \leqslant n\} \mapsto\{m \leqslant n\}$ such that $f(p)$ is a multiple of $a_{p}(n)+1$ for all primes $p \leqslant n$. Then $d(n !)$ is a divisor of $\prod_{p \leqslant n} f(p)$, which is a product of $\pi(n)$ distinct integers $\leqslant n$; hence, $d(n !)$ is a divisor of $n !$.

In order to define $f(p)$, we split the primes $p \leqslant n$ in three ranges.

CASE 1. $p \leqslant \sqrt{n} / 2$.

In this case, we take $f(p)=a_{p}(n)+1$. Clearly,

$$
a_{p}(n)+1 \leqslant \frac{n-1}{p-1}+1 \leqslant n \text { for all primes } p \geqslant 2 .
$$

To see that the numbers $f(p)$ are distinct for distinct primes $p$ in this range, assume that $q<p$ are both primes in $[2, \sqrt{n} / 2]$ and that $f(p)=f(q)$. Then the equation $f(p)=f(q)$ can be rewritten as

$$
\frac{n-s_{p}(n)}{p-1}=\frac{n-s_{q}(n)}{q-1}
$$

which yields

$$
\frac{n-s_{p}(n)}{n-s_{q}(n)}=\frac{p-1}{q-1}=1+\frac{p-q}{q-1} \geqslant 1+\frac{1}{q-1},
$$

which in turn implies that

$$
\frac{1}{q-1} \leqslant \frac{s_{q}(n)-s_{p}(n)}{n-s_{q}(n)} \leqslant \frac{s_{q}(n)-1}{n-s_{q}(n)} .
$$


Thus,

$$
\begin{aligned}
n & \leqslant s_{q}(n)+(q-1)\left(s_{q}(n)-1\right)=q s_{q}(n)-q+1<q s_{q}(n) \\
& \leqslant q(q-1)\left(\left\lfloor\frac{\log n}{\log q}\right\rfloor+1\right)<\frac{q^{2} \log (q n)}{\log q}<\frac{3 q^{2} \log n}{2 \log q}=\frac{3 q^{2} \log n}{\log \left(q^{2}\right)} .
\end{aligned}
$$

In the above chain of inequalities, we used aside from the right inequality (2.2) also the fact that $q<n^{1 / 2}$, therefore $q n<n^{3 / 2}$, so $\log (q n)<(3 / 2) \log n$. Since $4 \leqslant q^{2} \leqslant n / 4$, and the function $x \mapsto x / \log x$ is increasing for $x>e$, inequality (2.4) above yields

$$
\frac{n}{\log n}<\frac{3 q^{2}}{\log \left(q^{2}\right)} \leqslant \frac{3 n}{4 \log (n / 4)},
$$

leading to $\log (n / 4)<(3 / 4) \log n$, or $n / 4<n^{3 / 4}$, or $n<4^{4}=256$, which is not the case we are considering.

CASE 2. $\sqrt{n} / 2<p \leqslant n / 2$.

Let $p_{1}<p_{2}<\cdots$ be the increasing sequence of all prime numbers. Let $k:=\pi(\sqrt{n} / 2)$ and assume that $p_{k+1}, \ldots, p_{k+s}$ are all the primes in this case, where $k+s=\pi(n / 2)$. Observe that for such $p$, we have $p^{3}>n^{3 / 2} / 8>n$. Thus,

$$
a_{p}(n)=\left\lfloor\frac{n}{p}\right\rfloor+\left\lfloor\frac{n}{p^{2}}\right\rfloor
$$

and the second integer appearing on the right-hand side above is in $\{0,1,2,3\}$. We pick inductively $f\left(p_{k+i}\right)$ for $i=1, \ldots, s$ to be a positive integer in the interval $\mathcal{I}=[(n+2) / 2, n]$, satisfying the following properties

(i) it is distinct from $a_{2}(n)+1=n-s_{2}(n)+1$;

(ii) it is distinct from $f\left(p_{k+j}\right)$ for all $j=1, \ldots, i-1$;

(iii) it is a multiple of $a_{p_{k+i}}(n)+1$.

Observe that condition (i) says that $f\left(p_{k+i}\right) \neq f(2)=a_{2}(n)+1=n-s_{2}(n)+1$. To check that $f\left(p_{k+i}\right) \neq f(p)$ for all $p \in[3, \sqrt{n} / 2]$, observe that for such $p$ we have that

$f(p)=a_{p}(n)+1=\frac{n-s_{p}(n)}{p-1}+1 \leqslant \frac{n-1}{p-1}+1 \leqslant \frac{n-1}{2}+1=\frac{n+1}{2}<f\left(p_{k+i}\right)$.

To justify that we can choose $f\left(p_{k+i}\right)$ as in (i)-(iii) above, it suffices to show that the number of multiples of $a_{p_{k+i}}(n)+1$ in $[(n+2) / 2, n]$ exceeds $i$, since then one such multiple can be chosen to avoid the single number $n-s_{2}(n)+$ 1 appearing at (i), and the already chosen $i-1$ numbers $f\left(p_{k+j}\right)$ for $j=$ $1, \ldots, i-1$. Now since

$$
a_{p_{k+i}}(n)+1 \leqslant \frac{n-1}{p_{k+i}-1}+1=\frac{n+p_{k+i}-2}{p_{k+i}-1},
$$


we find that the number of integers multiples of $a_{p_{k+i}}(n)+1$ in $\mathcal{I}$ is at least

$$
\left\lfloor\frac{n-(n+2) / 2}{a_{p_{k+i}}(n)+1}\right\rfloor \geqslant\left\lfloor\frac{(n-2)\left(p_{k+i}-1\right)}{2\left(n+p_{k+i}-2\right)}\right\rfloor .
$$

So, it suffices to show that

$$
\frac{(n-2)\left(p_{k+i}-1\right)}{2\left(n+p_{k+i}-2\right)} \geqslant i+2 .
$$

The above inequality $(2.5)$ is equivalent to

$$
p_{k+i} \geqslant \frac{(n-2)(2 i+5)}{n-2(i+3)} .
$$

We first show that inequality

$$
\frac{n-2}{n-2(i+3)} \leqslant \frac{5}{4}
$$

holds. Inequality $(2.7)$ is equivalent to $i+2.2 \leqslant n / 10$. But clearly

$$
i+2.2 \leqslant \pi(n / 2)-\pi(\sqrt{n} / 2)+2.2 \leqslant \pi(n / 2),
$$

where the last inequality follows because $n \geqslant 100$, so $\sqrt{n} / 2 \geqslant 5$, so $\pi(\sqrt{n} / 2) \geqslant$ 3. Thus, we need that $\pi(n / 2) \leqslant n / 10$. By Theorem 2 on [7, Page 69], we have that

$$
\pi(n / 2)<\frac{n / 2}{\log (n / 2)-1.5}
$$

Thus, inequality (2.7) holds provided that

$$
\frac{n / 2}{\log (n / 2)-1.5} \leqslant \frac{n}{10},
$$

which is equivalent to $n>2 e^{6.5}$, which holds for $n \geqslant 1331$. Thus, inequality (2.7) holds, so in order for inequality (2.6) to hold, it is enough that

$$
p_{k+i} \geqslant \frac{5}{2}\left(i+\frac{5}{2}\right) \text {. }
$$

By inequality (3.12) on [7, Page 69], we have

$$
p_{k+i}>(k+i) \log (k+i)>(2.5+i) \log k,
$$

where the right-most inequality holds because $k=\pi(\sqrt{n} / 2)>2.5$. Thus, in order for inequality $(2.8)$ to hold, it suffices that $k \geqslant e^{2.5}$, or $k \geqslant 13$. Since $k=\pi(\sqrt{n} / 2)$, it suffices that $\sqrt{n} / 2 \geqslant p_{13}$, or $n \geqslant 2 p_{13}^{2}=3362$. In conclusion, since $n \geqslant 3400$, the inequality $(2.5)$ holds for all $i=1, \ldots, \pi(n / 2)-\pi(\sqrt{n} / 2)$, which takes case of the injection $f(p)$ in this case.

CASE 3. $n / 2<p \leqslant n$.

In this case, $a_{p}(n)+1=2$ for all such primes $p$. We assign to each prime $p$ a distinct even number in the interval $[(n+4) / 4, n / 2]$, except for the possibly 
even number $a_{3}(n)+1=\left(n-s_{3}(n)+2\right) / 2$. Observe that if $p$ is a prime in this case, then

$$
a_{2}(n)+1=n+1-s_{2}(n) \geqslant n+1-\left(\frac{\log n}{\log 2}+1\right)=n-\frac{\log n}{\log 2}>\frac{n}{2},
$$

so $f(p)$ is not $f(2)$. Also, $f(p)$ is not $f(3)$ by construction. If $q \geqslant 5$ is in Case 1 , then

$$
f(q)=a_{q}(n)+1=\frac{n-s_{q}(n)}{q-1}+1 \leqslant \frac{n-1}{q-1}+1 \leqslant \frac{n-1}{4}+1=\frac{n+3}{4}<f(p) .
$$

Finally, if $q$ is in Case 2 , then $f(q) \geqslant(n+2) / 2>f(p)$. Thus, in order to justify that one can define $f(p)$ in the above way for all primes $p \in(n / 2, n]$, it suffices to show that the interval $\mathcal{J}=[(n+4) / 4, n / 2]$ contains at least $\pi(n)-\pi(n / 2)+1$ even numbers. The number of even numbers in $\mathcal{J}$ is at least

Thus, we need to check that

$$
\left\lfloor\frac{n / 2-(n+4) / 4}{2}\right\rfloor=\left\lfloor\frac{n-4}{8}\right\rfloor \geqslant \frac{n-11}{8} .
$$

$$
\frac{n-11}{8} \geqslant \pi(n)-\pi(n / 2)+1 .
$$

By [7, Theorem 2], we have that both inequalities

$$
\pi(n)<\frac{n}{\log n-1.5} \quad \text { and } \quad \pi(n / 2)>\frac{n / 2}{\log (n / 2)-0.5}
$$

hold in our range for $n \geqslant 3400$. Hence, in order for (2.9) to hold it suffices, via inequalities (2.10), that the inequality

$$
\frac{n-11}{8}>\frac{n}{\log n-1.5}-\frac{n / 2}{\log (n / 2)-0.5}+1
$$

holds. This last inequality certainly holds for all $n \geqslant 3400$.

Thus, we have just showed that $d(n !)$ divides $n$ ! for all $n \geqslant 3400$, which completes the proof of this theorem.

\section{The Proof of Theorem 1.2}

First, some preliminaries. We let $\left\{L_{n}\right\}_{n} \geqslant 1$ be the Lucas companion of the Fibonacci sequence given by $L_{1}=1, L_{2}=3$ and $L_{n+2}=L_{n+1}+L_{n}$ for all $n \geqslant 1$. There are many identities relating Fibonacci and Lucas numbers, such as

$$
F_{2 n}=F_{n} L_{n}, \quad L_{n}^{2}-5 F_{n}^{2}=4(-1)^{n} \quad \text { and } \quad L_{3 n}=L_{n}\left(L_{n}^{2}-3(-1)^{n}\right)
$$

valid for all positive integers $n$. We shall freely use such identities in what follows. They can be easily shown to hold by using the Binet formulas

$$
F_{n}=\frac{\alpha^{n}-\beta^{n}}{\alpha-\beta} \quad \text { and } \quad L_{n}=\alpha^{n}+\beta^{n}
$$


valid for all $n \geqslant 1$, where $(\alpha, \beta):=\left(\frac{1+\sqrt{5}}{2}, \frac{1-\sqrt{5}}{2}\right)$ are the two roots of the characteristic equation $x^{2}-x-1=0$ of the sequence of Fibonacci (or Lucas) numbers.

We also use the well-known fact that $F_{n}$ is even if and only if $n$ is a multiple of 3. Furthermore, if $n=3 m$ with $m$ odd, then $2 \| F_{n}$, while if $n=2^{a} \cdot 3 m$ with some $a \geqslant 1$ and $m$ odd, then $2^{a+2} \| F_{n}$.

The main idea for this proof is that if a positive integer $m$ has the property that the exponent of 2 in the factorization of $d(m)$ is bounded above by some nonnegative integer $K$, then $m$ can have at most $K$ distinct primes appearing at odd exponents in its factorization. In particular, $m$ is a square when $K=0$. Throughout this proof, we use $\square$ for a square of an integer. It is well-known that the only positive integers $n$ such that $F_{n}$ is $\square$ or $2 \square$ are $n \in\{1,2,3,6,12\}$, and the only positive integers $n$ such that $L_{n}=\square$ or $2 \square$ are $n \in\{1,3,6\}$ (see $[3,4])$.

After the above preliminaries, we are ready to proceed with the proof of Theorem 1.2. We use a divide and conquer approach. We divide the set of potential $n$ such that $d\left(F_{n}\right) \mid F_{n}$ according to the exponent of 2 in the factorization of $F_{n}$.

(i) $F_{n}$ is odd. Then $d\left(F_{n}\right)$ is a divisor of $F_{n}$, so it is odd. Hence, $F_{n}=\square$, so $n \in\{1,2,12\}$. The only convenient solutions here are $n \in\{1,2\}$.

(ii) $2 \| F_{n}$. Then $n=3 m$, where $m$ is odd. Since $2 \| F_{n}$ and $d\left(F_{n}\right)$ can be a multiple of 2 but not of 4 , it follows that $F_{n}=2 \square$. Thus, $n \in\{3,6\}$, of which only the solution $n=3$ is convenient.

(iii) There is no $n$ such that $4 \| F_{n}$.

(iv) $8 \| F_{n}$. Then $d(8)=4$ divides $d\left(F_{n}\right)$, a number which may be divisible by 8 but not by 16 . We then get that $F_{n}=8 \delta \square$, where $\delta \in\{1, p\}$ and $p$ is some odd prime. Furthermore, $n=6 m$ with $m$ odd. Then $F_{n}=$ $F_{3 m} L_{3 m}$ by the first of the relations (3.1), and the greatest common divisor of $F_{3 m}$ and $L_{3 m}$ is 2 by the second of the relations (3.1). More precisely, $2 \| F_{3 m}$ and $4 \| L_{3 m}$. Now the equation $F_{2 m} L_{3 m}=8 \delta \square$ implies that either $F_{3 m}=2 \square$, or $L_{3 m}=\square$, both of which giving $m \in\{1,2\}$, of which only $m=1$, leading to $n=6$ is a convenient solution.

(v) $16 \| F_{n}$. Then $n=12 m$, where $m$ is odd. Furthermore $d\left(F_{n}\right)$ is a multiple of $d(16)=5$, so $5 \mid F_{n}$, therefore $5 \mid m$. Hence, $n$ is a multiple of 60 . Suppose first that $n=2^{4} \cdot 3^{b} \cdot 5^{c}$ with some positive integers $b$ and $c$. Then $F_{60} \mid F_{n}$, and $F_{60}$ has five prime factors $p>5$ each one of them appearing with exponent one in its factorization, namely $p \in\{11,31,41,61,2521\}$. Since all prime factors of $n$ are $\leqslant 5$, it follows that each of these five primes appears with exponent one in the factorization of $F_{n}$. Hence, $2^{5}\left|d\left(F_{n}\right)\right| F_{n}$, which is a contradiction. Thus, $n$ must have at least a prime factor exceeding 5 , 
and, in particular, $\omega(n) \geqslant 4$, where, as usual, for a positive integer $t$ we write $\omega(t)$ for the number of distinct prime factors of $t$. Write

$$
F_{n}=F_{12 m}=F_{3 m} L_{3 m} L_{6 m} .
$$

The above relation follows by applying the first of relations (3.1) twice, once for $n=12 m$, and once for $n / 2=6 m$. The greatest common divisor of any two of the three factors from the right-hand side of relation (3.2) above is 2 . [2, Lemma 3] shows that $F_{3 m}$ has at least $\omega(3 m) \geqslant 3$ distinct odd prime factors appearing in its factorization at an odd exponent. If $L_{3 m}=\square$ or $2 \square$, we then get $m \in\{1,2\}$, so $n \in\{12,24\}$, and none leads to a convenient solution. So, $L_{3 m}$ has (at least) an odd prime factor appearing at an odd exponent in its factorization. Similarly, if $L_{6 m}=\square$ or $2 \square$, then $m=1$, leading to $n=12$, which is not convenient. Thus, $L_{6 m}$ also has (at least) an odd prime factor appearing at an odd exponent in its factorization. But this shows that $F_{n}$ has at least five prime factors appearing at an odd exponent in its factorization, so $2^{5}\left|d\left(F_{n}\right)\right| F_{n}$, which is a contradiction.

From now on, we assume that $a \geqslant 5$ is such that $2^{a} \| F_{n}$. Then $n=$ $2^{a-2} \cdot 3 m$, where $m$ is odd. To continue, we need the following lemma.

Lemma 3.1. Let $m=12 k$, where $k$ is a positive integer. Then $L_{m}$ has at least two odd primes appearing with odd exponent in its prime factorization.

Proof. Assume that this is not so. Note that $2 \| L_{m}$. Then $L_{m}=2 \delta \square$, where $\delta \in\{1, p\}$ with $p$ a prime. We use the formula $L_{12 k}=L_{4 k}\left(L_{4 k}^{2}-3\right)$, which is the third of the formulae (3.1) with $n=4 k$. The two factors on the right of the previous equality are coprime, for if $q$ is some common prime factor of them, then $q \mid L_{4 k}$ and $q \mid L_{4 k}^{2}-3$, so $q \mid 3$, therefore $q=3$. Hence, $3 \mid L_{4 k}$, which is false because the only numbers of the form $L_{t}$ which are multiples of 3 are for $t \equiv 2(\bmod 4)$. Thus, from $L_{4 k}\left(L_{4 k}^{2}-2\right)=2 \delta \square$, we get that either $L_{4 k}=\square$ or $2 \square$, or $L_{4 k}^{2}-3=\square$, or $2 \square$. None of the two equations of the first possibility can hold by the results from $[3,4]$. As for the pair of equations of the second possibility, observe that the first one leads to a positive integer solution $(x, y)$ of the equation $x^{2}-3=y^{2}$, or $(x-y)(x+y)=3$, whose only solution is $(x, y)=(2,1)$, which is not convenient because $L_{4 k}>2$, whereas the second one leads to a positive integer solution $(x, y)$ of the equation $x^{2}-3=2 y^{2}$, which reduced modulo 3 gives $x^{2} \equiv 2(\bmod 3)$, which is also impossible. This completes the proof of the lemma.

We continue the proof of Theorem 1.2. We assume next that $m=1$, so $n=2^{a-2} \cdot 3$ for some $a \geqslant 5$. One can check that both $a=5$ and $a=6$ for which $n=24$ and $n=48$, respectively, are convenient solutions to our problem, but that $a=7$ and $a=8$ for which $n=96$ and $n=192$, respectively, 
are not convenient solutions. For $a \geqslant 9$, write

$$
F_{n}=F_{3} L_{6} L_{12} L_{24} \cdots L_{2^{a-3} \cdot 3},
$$

by repeated applications of the first relation (3.1). The greatest common divisor of any two factors appearing in the right-hand side of the above relation (3.3) is 2. The number $L_{2^{i} \cdot 3}$ has at least two odd prime factors appearing at odd exponents in its factorization; hence in the factorization of $F_{n}$, for all $i=2, \ldots, a-3$, by Lemma 3.1. Thus, $F_{n}$ has at least $2(a-4)=2 a-8>a$ odd distinct primes appearing at odd exponents in its factorization. Hence, $d\left(F_{n}\right)$ is divisible with $2^{a+1}$, a contradiction.

Next assume that $n=2^{a-2} \cdot 3 m$, where $m>1$ is odd. Now write

$$
F_{n}=F_{3 m} L_{3 m} L_{6 m} L_{12 m} \cdots L_{2^{a-3} m}
$$

again by repeated applications of the first relation (3.1). Again any two of the factors appearing on the right-hand side of the above relation (3.4) have greatest common divisor 2. By Lemma 3.1, the numbers $L_{2^{i} \cdot 3 m}$ have each at least two odd primes appearing at odd exponents in their factorization; hence in the factorization of $F_{n}$. Further, none of $F_{3 m}, L_{3 m}$, and $L_{6 m}$ is of the form $\square$ or $2 \square$ because $m>1$ is odd. Hence, each one of these three numbers has at least one odd prime appearing at an odd exponent in its factorization; hence in the factorization of $F_{n}$. Thus, $F_{n}$ has at least $3+2(a-4)=2 a-5$ odd prime factors appearing at odd exponents in the factorization of $F_{n}$. If $a>5$, then $2 a-5>a$, so $d\left(F_{n}\right)$ is divisible by $2^{a+1}$, a contradiction. If $a=5$, then $2 a-5=5$, but in this case also the prime 2 appears with an odd exponent in the factorization of $F_{n}$ (namely with the exponent 5 ), so in fact $d\left(F_{n}\right)$ is divisible by $2^{6}$, again a contradiction.

This finishes the proof of Theorem 1.2.

\section{ACKNOWLEDGEMENTS}

We thank the referee for comments which improved the quality of this paper. This work started during a visit of P. T. Y. at the School of Mathematics of the University of the Witwatersrand in May of 2011. He thanks the people of this department for their hospitality and support. This paper was written in Spring of 2011 while F. L. was on sabbatical from the Mathematical Institute UNAM from January 1 to June 30, 2011 and supported by a PASPA fellowship from DGAPA.

\section{REFERENCES}

[1] D. Baczkowski, M. Filaseta, F. Luca and O. Trifonov, On rational values of $\tau(n !) / m$ !, $\phi(n !) / m$ ! and $\sigma(n !) / m$ !, Int. J. Number Theory 6 (2010), 1199-1214.

[2] K. Broughan, M. González, R. Lewis, F. Luca, V. J. Mejía Huguet and A. Togbé, There are no multiply-perfect Fibonacci numbers, Integers 11A (2011), A7.

[3] J. H. E. Cohn, Fibonacci square numbers, etc., Fibonacci Quarterly 2 (1964), 109-113.

[4] J. H. E. Cohn, Lucas and Fibonacci numbers and some Diophantine equations, Proc. Glasgow Math. Assoc. 7 (1965), 24-28. 
[5] P. Erdős, S. W. Graham, A. Ivić and C. Pomerance, On the divisors of $n$ !, in Analytic Number Theory, Proceedings of a Conference in Honor of Heini Halberstam, Vol. 1, B. Berndt, H. Diamond, A. Hildebrand, eds., Birkhauser, Boston, 1996, 337-355.

[6] F. Luca, Equations involving arithmetic functions of factorials, Divulg. Mat. 8 (2000), $15-23$.

[7] J. B. Rosser and L. Schoenfeld, Approximate formulas for some func- tions of prime numbers, Illinois J. Math. 6 (1962), 64-94.

[8] C. Spiro, How often is the number of divisors of $n$ a divisor of $n$ ?, J. Number Theory 21 (1985), 81-100.

F. Luca

Instituto de Matemáticas

Universidad Nacional Autónoma de México

C.P. 58089, Morelia, Michoacán

México

and

The John Knopfmacher Centre

for Applicable Analysis and Number Theory

University of the Witwatersrand

P.O. Wits 2050

South Africa

E-mail: fluca@matmor.unam.mx

P. T. Young

Department of Mathematics

College of Charleston

Charleston, SC 29424

USA

E-mail: paul@math.cofc.edu

Received: 12.9.2011.

Revised: 16.11.2011. 